\title{
Analysis of Spring-in for Composite Plates Using ANSYS Composite Cure Simulation
}

\author{
AMEYA PATIL, REZA MOHEIMANI, TALAL SHAKHFEH \\ and HAMID DALIR
}

\begin{abstract}
Process induced dimensional changes in composite parts has been the topic of interest for many researchers. The residual stresses that develop in fiber-reinforced laminates during curing process while the laminate is confined to the process tool often leads to dimensional changes such as spring-in of angles and warpage of flat sections. Many experimental studies have put emphasis on this issue and various researches show different methods to predict these dimensional changes. The traditional trial-and-error approach can work for simple geometries, but composite parts with complex shapes require more sophisticated models. When composite laminates are subjected to thermal stresses, such as the heating and cooling processes during curing, they can become distorted as the difference between the in-plane and the through-thickness thermal expansion coefficient, as well as chemical shrinkage of the epoxy, causes the enclosed angle of curved sections and angle components to be reduced. Distorted components can cause problems during assembly, significantly increasing production costs and affecting performance. This paper focuses on predicting these shape deformations using software simulation of composite manufacturing and curing. Various factors such as resin shrinkage, degrees of cure, difference between coefficient of thermal expansion of mold and composite are taken into consideration. A cure kinetic model is presented which illustrates the matrix behavior during cure.
\end{abstract}

\section{BACKGROUND}

The necessity of lightweight design in aerospace and motorsports industry has increased the demand of carbon fiber reinforced plastics composite materials. Over the years composite material manufacturing has been growing progressively. Currently, process induced residual stresses and shape deformations of are still a big challenge for the manufacturers, as those unwanted distortions from original shape lead to bigger scrap rates and/or further difficulties during the assembly process. An assembly of deformed parts can cause a massive increase of the part's internal residual stress level which weaken the part's performance.

Ameya Patil, Hamid Dalir, Department of Mechanical and Energy Engineering, Purdue School of Engineering and Technology, Indianapolis, IN, USA.

Reza Moheimani, School of Mechanical Engineering, Purdue University, West Lafayette, IN, USA.

Talal Shakfeh, Senior FEA Engineering Analyst, Simutech Group Inc.-Toronto, 50 Ronson Drive, Suite 165 Toronto, Ontario M9W 1 B3 Canada 
Processe-induced distortions of small composite parts are not as critical as those of larger and complex parts. Nowadays, tool designers technically rely on their own experience to deal with the problem, often applying a trial-and-error approach to estimate process-induced deformations. While this gives reasonably decent results for parts with comparatively modest geometry, composite parts with sophisticated profiles require well-designed models to capture the interactions between different geometrical features. The most common problem found, using a standard factor of approximation, is that the spring-in varies due to various parameters. Therefore, standard approximation may not be an effective technique every time.

The need of having a reliable approach for predicting these process induced shape distortions has driven researchers to develop new methods. As composite materials are anisotropic in nature, the study of directional properties becomes important while predicting the final material properties. The fiber and matrix behavior during the manufacturing needs a comprehensive study for predicting the final shape of any composite laminate. Curing process analysis is the buidling block of obtaining the accurate results for predicting the final shape of the composite part.

Using Classical Laminate Theory (CLT), through thickness coefficient of thermal expansion can be obtained. Different analytical expressions have been proposed previously to estimate the dimensional changes in the laminate [1-6]. However, using the same expressions may not work every time as those expressions are developed based on a generalized approximation of the various factors affecting the shape distortions [7,8]. Simulating the entire curing process can be an effective method for expecting the final shape of the composite part.

Residual stresses that are unavoidably generated in composites while manufacturing, subsequently resluts in distortion of the final cured parts. These stresses get built up on fibermatrix, lamina-laminate, and structural levels. Generation of residual stresses can be attributed to several thermal mechanisms [9-14]. Inherent anisotropy of composite materials is the prominent one. The coefficient of thermal expansion (CTE) changes in different directions for composite materials. Previous experimental studies show that CTE of resin-dominated directions is much higher than that of fiber-dominated directions $[10,13]$. The dimensional instability, caused due to the very difference, results in generation of residual stresses. When the composite part is cooled, the residual stresses generated are compensated by the tool when the part is in contact with the tool. The part gets distorted to its equilibrium state to balance the internal residual stresses when the tool is removed.

Resin cure shrinkage is also significant in generation of residual stresses [11]. For polymeric composites, the resin shrinks when polymerization and cross-linking reactions take place, causing a volume drop and consequently material density increses. Shrinkage is more evident in resin dominated directions of the material $[7,10]$. The induced stresses which grow throughout cure due to CTE mismatch and resin shrinkage are capable of causing distortion and premature cracking of the composite laminates. Effect of moisture is also an important aspect for analyzing the internal stresses induced by cyclical hygrothermal conditions [15].

Another factor that is responsible for generation of residual stresses is tool-part interaction. Owing to the difference in CTE of tool and that of ply in proximity with tool, the tool and the ply expand at different rate upon heating. This mismatch in expansion is responsible for the residual stresses induced in outer plies of the composite laminate. The frictional forces on the 
ply in contact with tool cause uneven distribution of thermal strains across the laminate. Upon tool removal this effect can be observed as a distortion in the final part [16-18].

Reduction in strength and shape distortions are the prime effects of residual stress. Strength of the component is affected by stresses at the fiber-matrix, lamina-laminate, and structural levels all affect whereas dimensional fidelity is affected by only lamina-laminate and structural level stresses to any significant step [7]. Therefore, predicting residual stresses generated in the composite part is essential.

Table 1 shows the classification of parameters responsible for generation of residual stresses. This sorting offers sources of residual stresses associated to material selection and part design to be separated from sources that are inspected by processing.

TABLE 1. CLASSIFICATION OF THE PARAMETERS RESPONSIBLE FOR DIMENSIONAL CHANGES IN COMPOSITE PART.

\begin{tabular}{|c|c|}
\hline $\begin{array}{c}\text { Intrinsic } \\
\text { Parameters }\end{array}$ & Extrinsic Parameters \\
\hline Part Angles & Tool material \\
\hline Thickness & Curing Pressure and Temperature \\
\hline Lay-up & Curing Time \\
\hline Flange Length & Tool-part interaction \\
\hline
\end{tabular}

Tool dimensions are required to be modified to compensate for process induced distortions. Simulating the composite curing process can help to predict spring-in for composite parts before the first physical prototype is produced, reducing process development costs and increasing product quality. Using simulation, it becomes easier and more reliable to foresee the tooling geometry needed to steadily produce high-quality structures within fitted dimensional tolerances. Simulation can also help predicting the shape deformations in complex and large shaped composite parts, accurately. The thermo-mechanical simulation of the curing process can provide the accurate final part shape.

This paper aims to present a reliable approach to estimate accurate final shape of the composite part which can be used later as an input to compensate during tool design. While few methods presented structural finite element methods for prediction of residual stresses [1-9], the other methods $[10,12,18]$ put emphasis on thermal analysis to analyze the stress generation. The method presented here, however, takes both thermal and structural aspects of curing process into account. The ability to obtain the thermo-mechanical results makes the methodology unique from the other previous methods. The simulation of curing process is an easy, time saving and cost saving method. The focus on prediction of residual stresses enables one to address it while manufacturing.

\section{SIMULATION PROCESS}

ANSYS Composite Cure Simulation (ACCS) was used to analyze the residual stresses and spring in composite parts. The ACCS is completely combined into the Workbench setting. The required composite material data for the cure simulation is fed in the engineering data unit. 
The connectivity of ACCS to ANSYS Composite PrepPost, similar to other ANSYS products, enables continuous exchange of simulation and process design data. The connection with ANSYS DesignXplorer allows progressive design optimization of material and manufacturing process constraints [19].

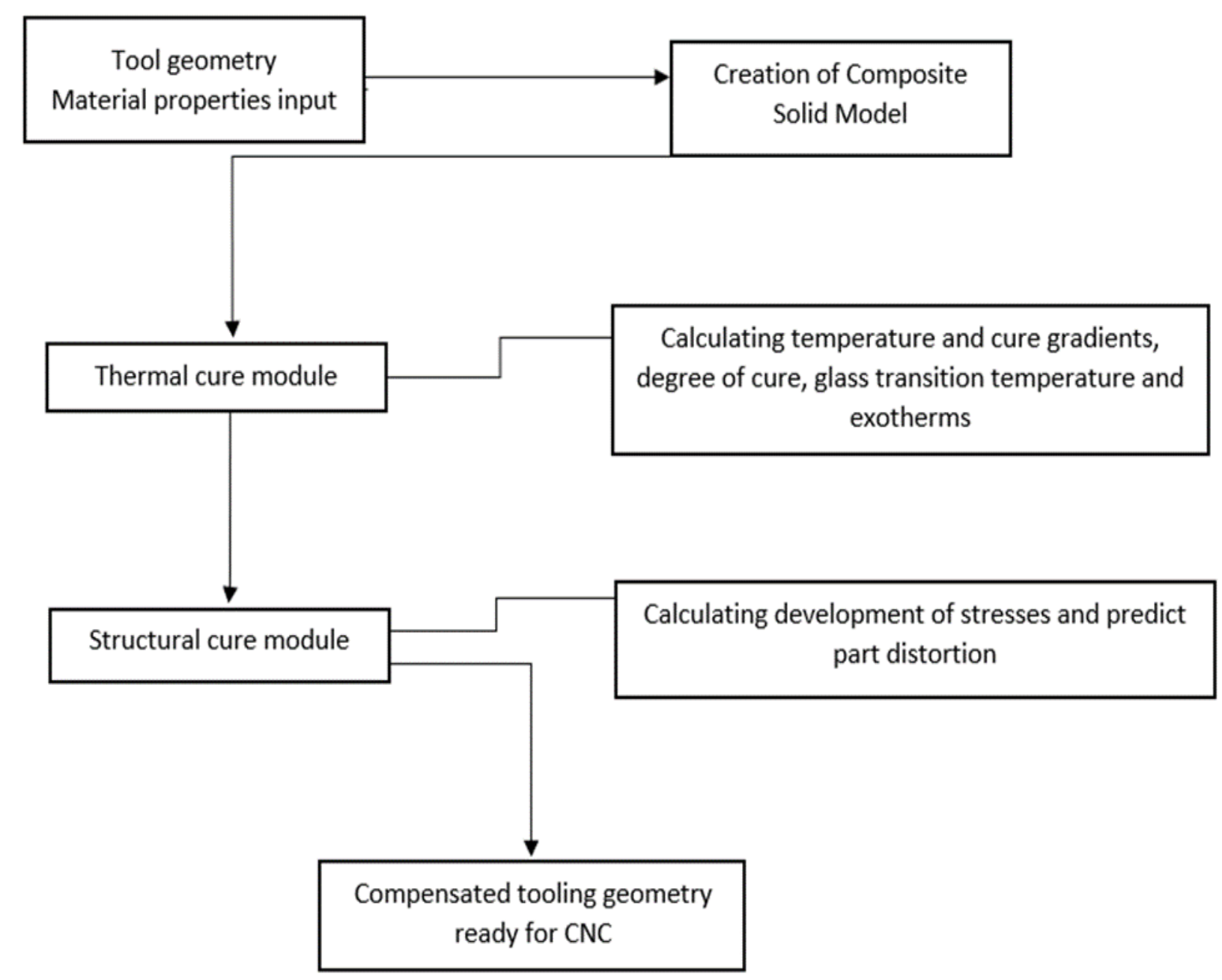

Figure 1. ACCS Simulation Workflow.

The ACCS chemical solver is being applied within the transient thermal module and simulation for development of polymerization and glass transition temperature, similarly internal heat generation related to exothermic cross-linking reactions has been conducted. The thermal and cure data then is fed into the structural data unit where the ACCS cure material model studies progress of residual stresses and process-induced distortions. ACCS utilizes a fast threestep simulation approach, for comparatively thin laminates ( $<5 \mathrm{~mm}$ thick), where an even temperature distribution is assumed. This quick solution can be applied in the early design stages for fast assessment of the process parameters. Upon completion of simulation procedure, the distorted geometry of FE model is able to generate compensated tooling geometry [19]. ACCS simulation workflow has been elaborated in figure. 1 . 


\section{PROCESS MODELLING}

To obtain a through thickness cure properties the analysis must be done on a solid composite model. A 3-D finite element solid model was used for this analysis. A shell model was first extracted from the CAD file created using Autodesk FUSION 360. The L-shaped plate (Fig. 2 (a)) was then imported to ANSYS Space-claim platform for creating the shell model. ANSYS Composite Prep-post module was then used to create a composite solid model.

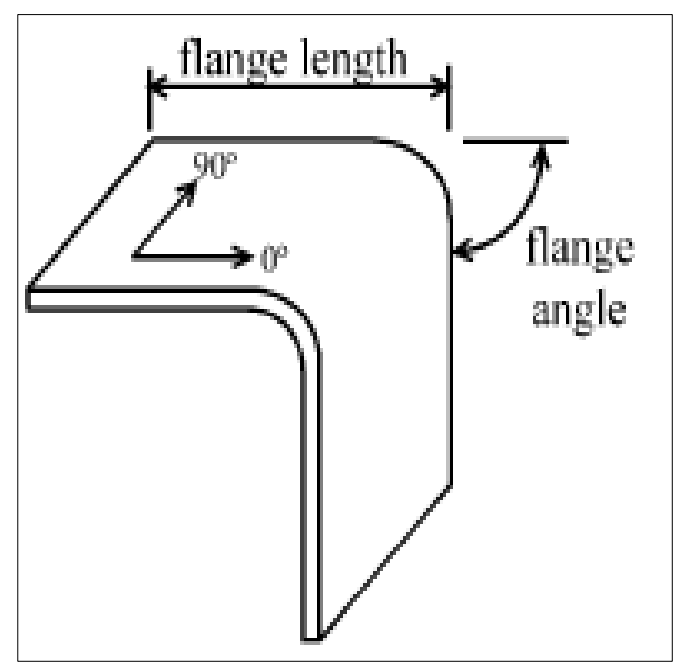

Figure 2. (a) Composite lay-up sequences.

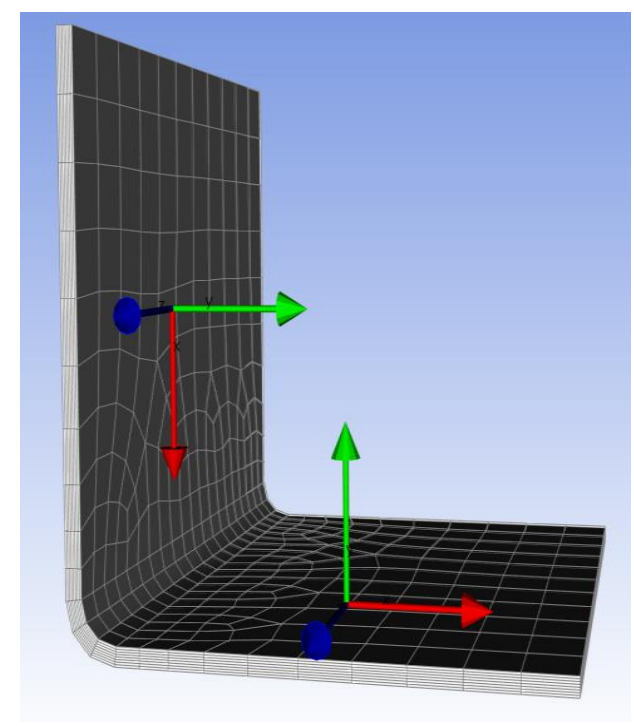

Figure 2. (b) Composite Solid model of L-shaped plate.

Mesh generation is a significant step in any FE simulation. In FE stress analysis knowing whether key stresses converge and checking if they have converged to a rational level of precision is important. A suitable mesh must be used with respect to the shape and size of the elements to obtain results that are consistent when using FEM. The solution accuracy is usually linked with mesh quality and mesh density [20]. FEA convergence defines the connection between the number of elements or degree of freedom and the analysis accuracy. Meshing was performed (Fig. 2 (b)) on the geometry to get accurate results. [23-25]

Hexcel AS4-8552 was the selected curable material used inside ANSYS. Hexcel AS48552 is a unidirectional prepreg with a high-performance tough epoxy matrix. This material is widely used in aerospace structures. It shows good impact resistance and damage tolerance for a widespread range of applications. Hexcel AS4-8552 shows good translation of fiber properties. For simulating the entire curing process, it is necessary to have the all the orthotropic properties available during the analysis. The cure kinetic equations are then used to obtain the results such as DOC, material state, glass transition temperature and heat of reaction. Table 2. illustrates all the material properties associated with Hexcel AS4-8552. The material properties presented in table are pre-defined inside ANSYS. It is possible to input material data inside ANSYS for different materials having different properties in $\mathrm{X}, \mathrm{Y}$ and $\mathrm{Z}$ directions. 
TABLE 2. MATERIAL PROPERTIES FOR HEXCEL AS4-8552.

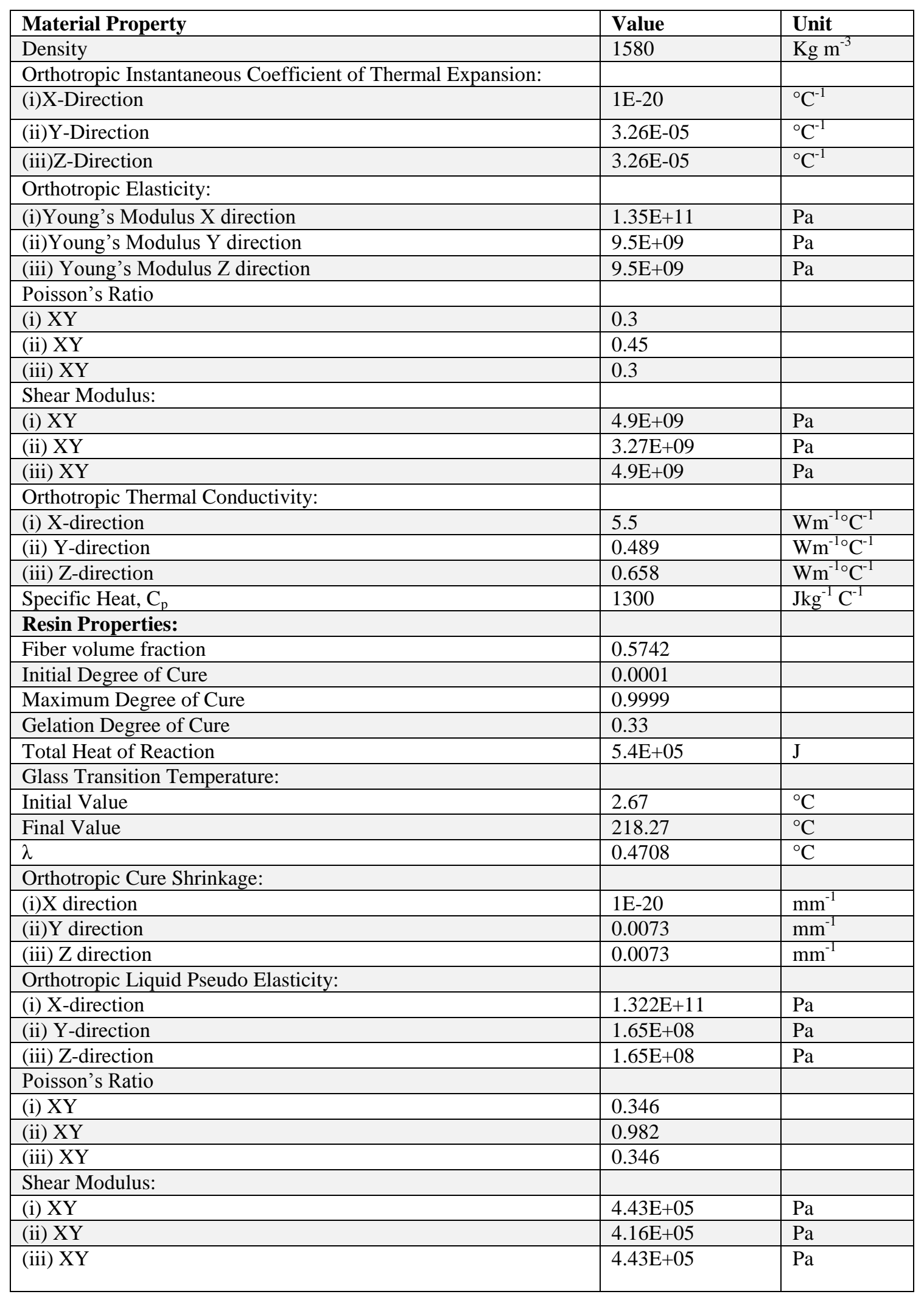




\section{CURE KINETIC MODEL}

The resin characterization is an important factor in the manufacturing of composite materials. Resin processing properties and their associated constitutive models are necessary as to describe and optimize the processing parameters and predict the final properties of a composite structure. The simulation results are based on cure kinetic model presented by researchers where the case study on CYCOM 890RTM Epoxy Resin was conducted to investigate thermomechanical behavior of the resin [21].

The total heat of reaction released during the cure dynamic was measured using modulated differential scanning calorimeter (MDSC), whereas isothermal scans were used to monitor the heat flow during a series of isothermal cures. The measured heat created by the resin was then converted into cure rate by the assumption that the rate of reaction, $\mathrm{d} \alpha / \mathrm{dt}$, is proportional to the rate of the heat flow, $\mathrm{dH} / \mathrm{dt}$ :

$$
\frac{d \alpha}{d t}=\frac{1}{H_{T}} \frac{d H}{d t}
$$

where $\mathrm{H}_{\mathrm{T}}$ is the overall exothermic heat of reaction. The degree-of-cure of the resin can be gained by taking integral of the area under the curve of cure rate vs time [21].

$$
\alpha=\frac{1}{H_{T}} \int_{0}^{1}\left(\frac{d H}{d t}\right) d t
$$

Thus, the cure rate can be stated as a function of the degree-of-cure and compared to existing cure kinetics models. The autocatalytic cure model with a diffusion factor developed by Hubert et al. [5], is by the following equation:

$$
\frac{d \alpha}{d t}=K \frac{\alpha^{m}(1-\alpha)^{n}}{1+\exp \left[C\left(\alpha-\left(\alpha_{C 0}+\alpha_{C T} T\right)\right)\right]}
$$

where $\mathrm{K}$ is a rate constant resulting an Arrhenius temperature dependency [21].

$$
K=A \exp \left(\frac{-E_{A}}{R T}\right)
$$

The autocatalytic model (Equation (4)) with the following constant values, $\mathrm{A}=58528 \mathrm{~s}^{-1}$, $\mathrm{Ea}=68976 \mathrm{~J} / \mathrm{mol}, \mathrm{n}=0.6, \mathrm{~m}=0.63, \mathrm{C}=15.66, \alpha_{\mathrm{C} 0}=-0.90$ and $\alpha_{\mathrm{CT}}=0.0039 \mathrm{~K}^{-1}$ accurately offers the resin cure evolution for the different dynamic and isothermal cases addressed [21].

The glass transition temperature ( $\mathrm{Tg}$ ) knowingly affects the resin mechanical properties , changing from its rubbery to glassy state. An increase in the CTE is noticed when the resin progresses from its glassy state to its rubbery state with a temperature ramp. The evolution of the Tg with the degree-of-cure was modeled with the DiBenedetto equation [21].

$$
\frac{T_{g}-T_{g 0}}{T_{g \infty}-T_{g 0}}=\frac{\lambda \alpha}{1-(1-\lambda) \alpha}
$$


where $T g$ is the glass transition temperature, $T_{g 0}$ and $T_{g \infty}$ are the glass transition temperatures of uncured and fully cured resin, respectively, $\alpha$ is the degree-of-cure and $l$ is a constant used as a fitting parameter valued between 0 and 1.

\section{THERMAL RESULTS}

The thermal non-linear simulation results were obtained using a semi-empirical model. Figures 3 and figure 4 shows the results for transient thermal solution of the composite curing simulation. Thermal analysis gives the results for Degree of Cure (DOC), Glass Transition Temperature and material state. Effect of cure shrinkage during the polymerization process is also considered during the thermal analysis.

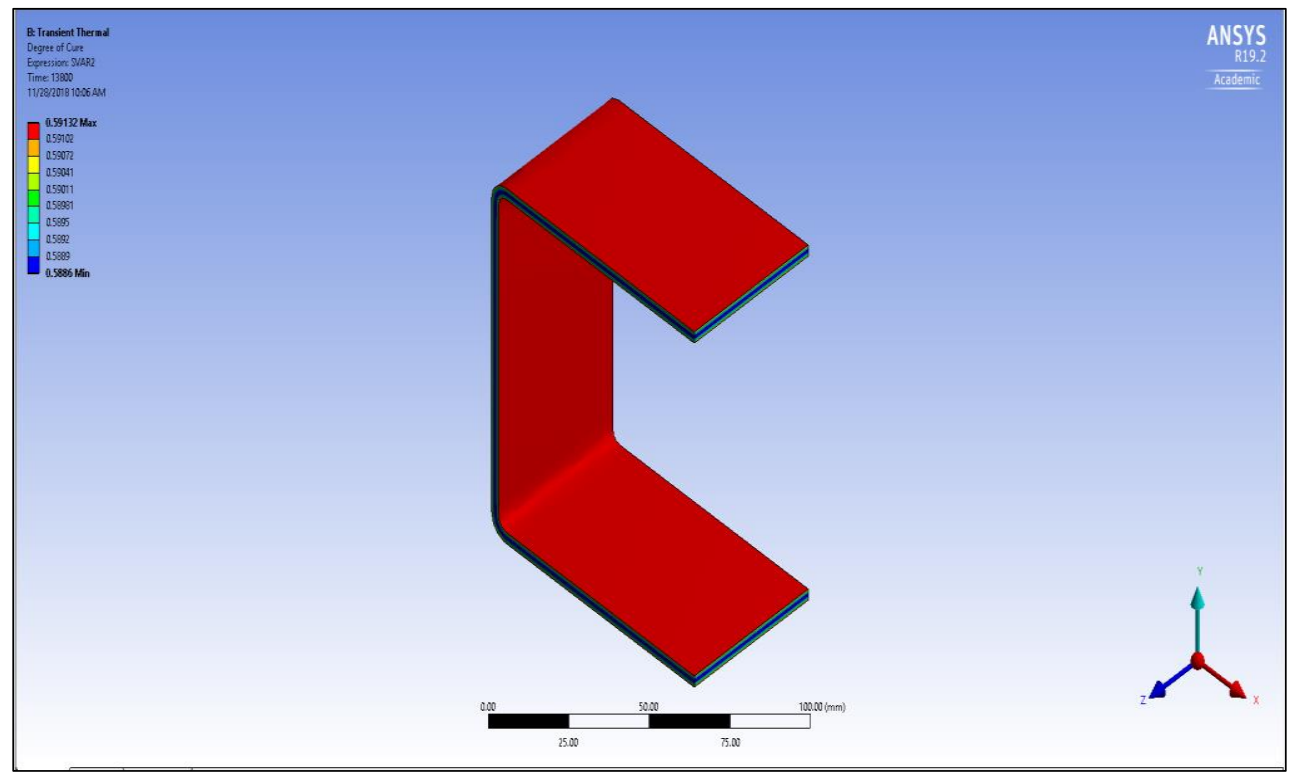

Figure 3. Degree of Cure Contour Results.

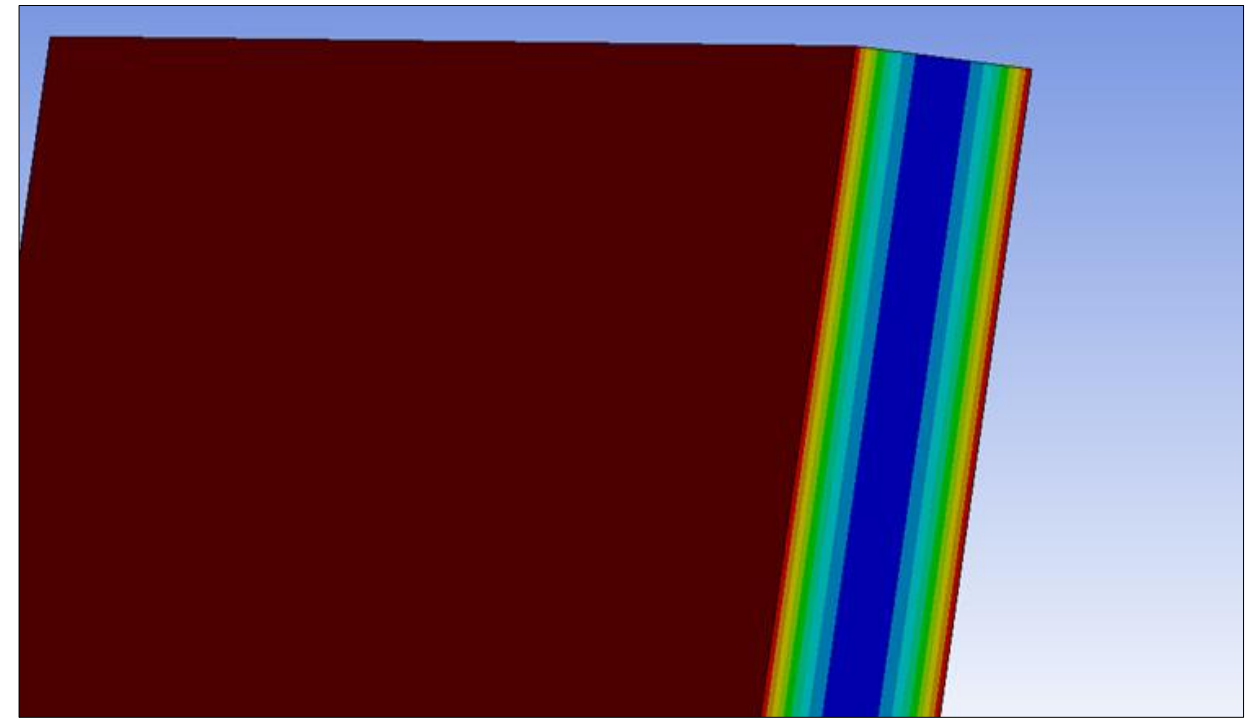

Figure 4. Ply-wise Degree of Cure Results. 
Degree of cure at any point of the curing process can be obtained using this simulation. Figure 5 subsequently represents the table of degree of cure at each time step during the simulation. The degree of cure numbers is obtained directly from the software which is the advantage of this method over previous methods. Earlier for obtaining DOC values it was necessary to use Differential Scanning Calorimeter (DSC) while conducting the experiments. A plot is then plotted (figure 6) to present the data of DOC vs Time.

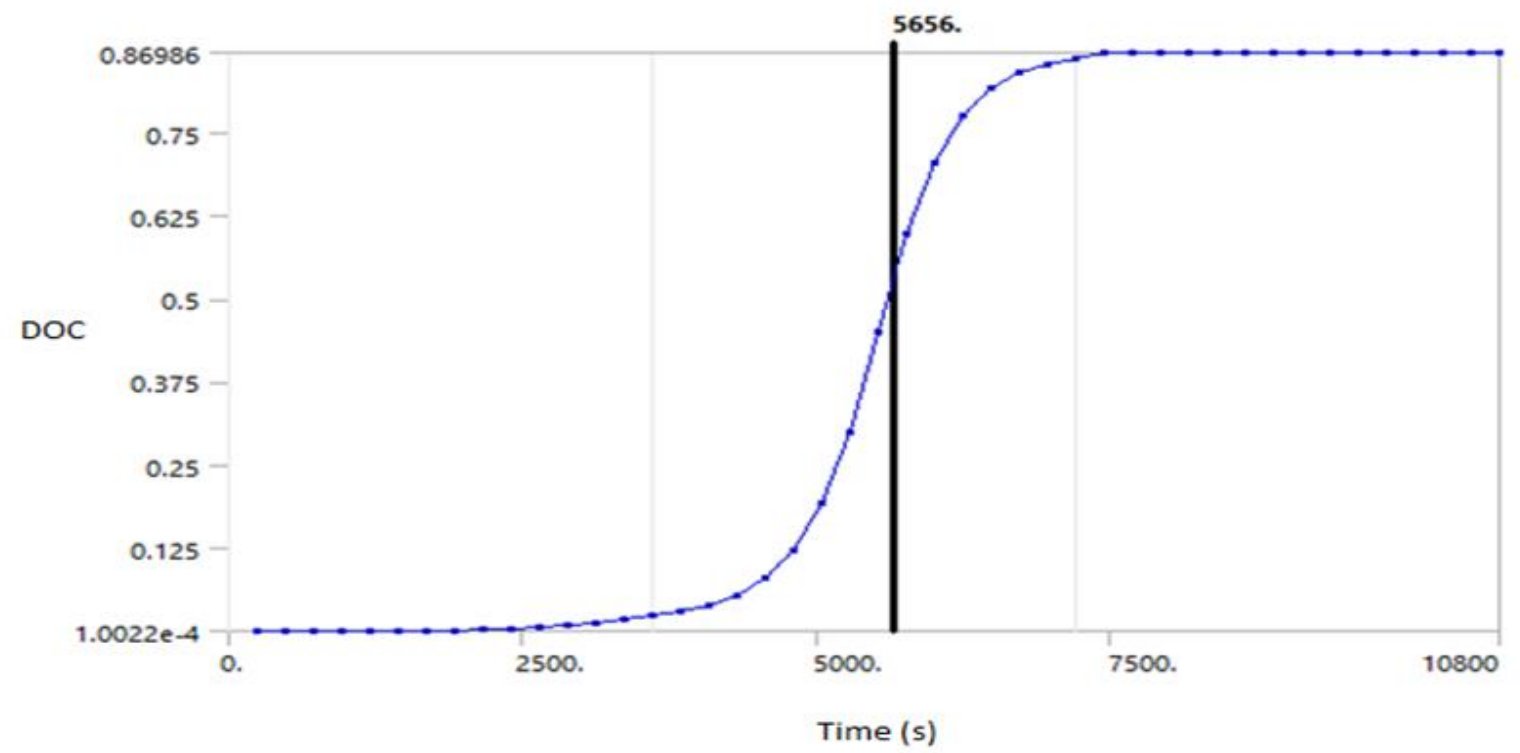

Figure 5. Plot of degree of cure vs time.

Figure 6 presents the cure cycle applied to the composite part inside the software. A twohold cure cycle where the part was heated from room temperature to $120^{\circ} \mathrm{C}$, held for half hour and then again heated to $180^{\circ} \mathrm{C}$ and again held at the same temperature for half hour and then was cooled down to the room temperature.

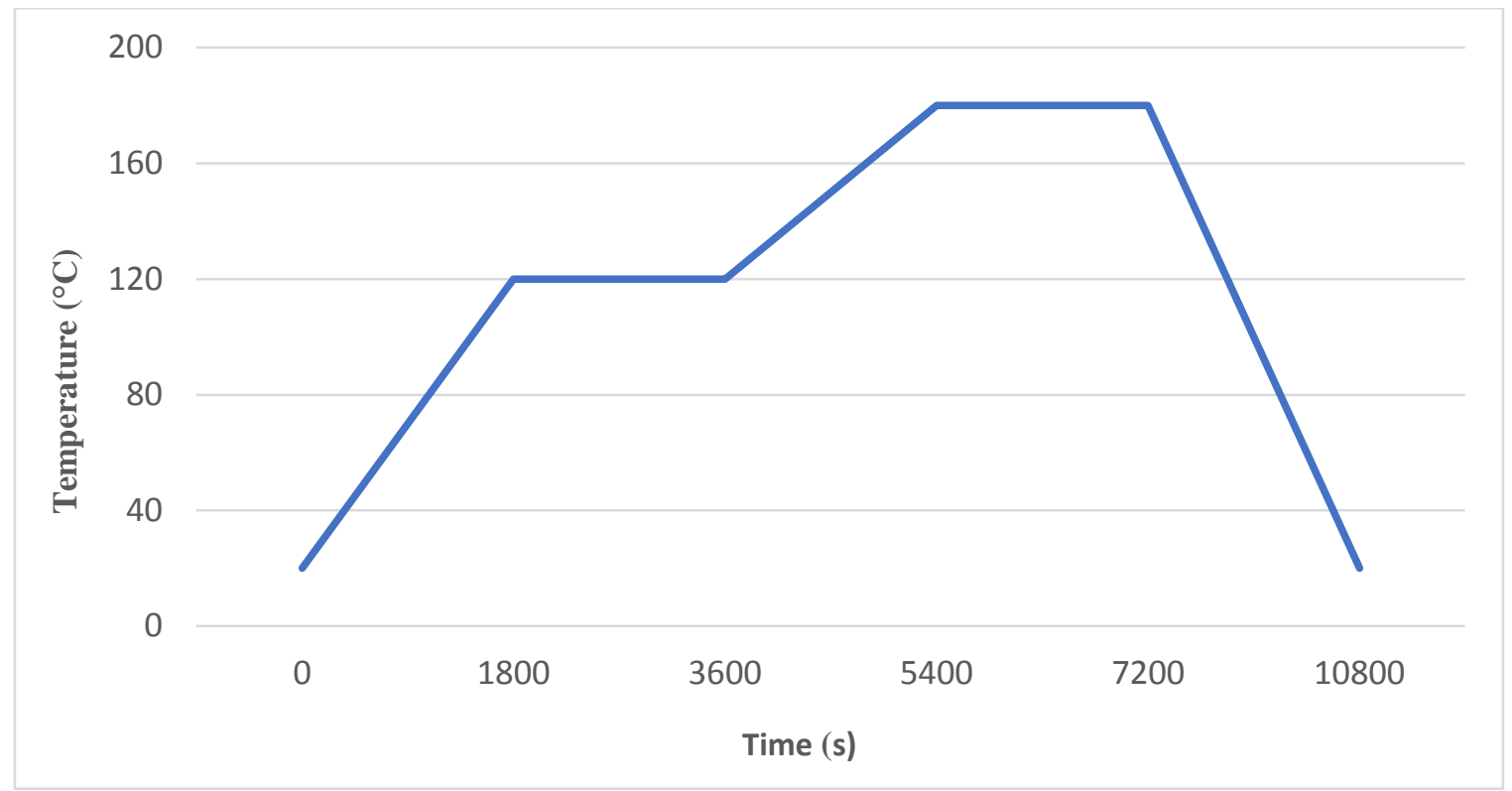

Figure 6. Schematic of the Two-hold cure cycle, Temperature vs. time. 
Figure 7 displays the progression of glass transition temperature $\left(\mathrm{T}_{\mathrm{g}}\right)$ throughout the cure cycle (two-hold). From these results the phase change of the resin can be analyzed. The resin converts from liquid phase to a rubbery phase when gelation occurs. The resin then converts to a glassy solid state. The glass transition temperatures at both these phase changes can be obtained from these results which help in predicting the material phase at any specific point during the cure cycle.

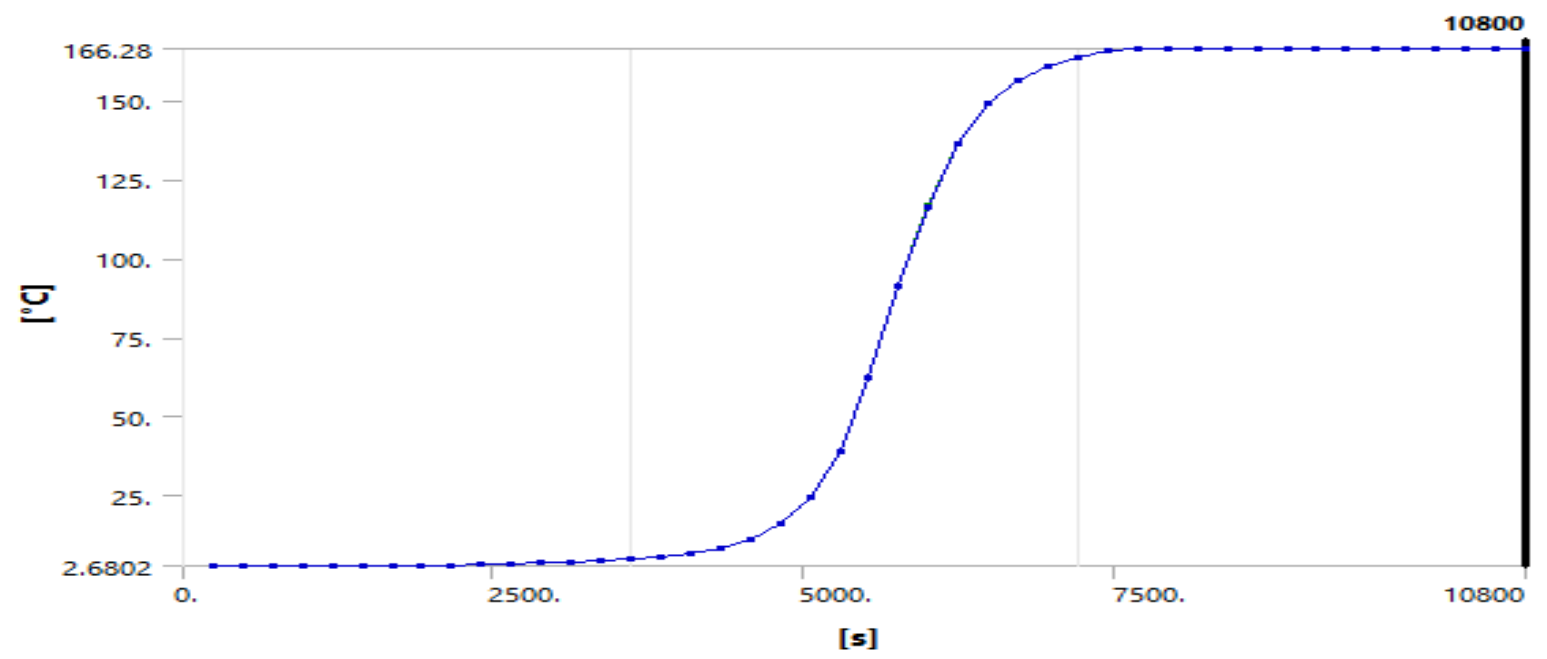

Figure 7. $\mathrm{T}_{\mathrm{g}}$ vs time plot for two-hold cure cycle.

\section{STRUCTURAL RESULTS}

The thermal model is then exported to the static structural module to obtain the results for process induced shape deformations and residual stresses. The thermal loads obtained act as initial boundary conditions for the analysis. Additional structural boundary conditions are applied to the part as well. The constraint (frictionless support) at the outer surface acts a tool for the composite part. The support remover tool simulates the tool removal process.

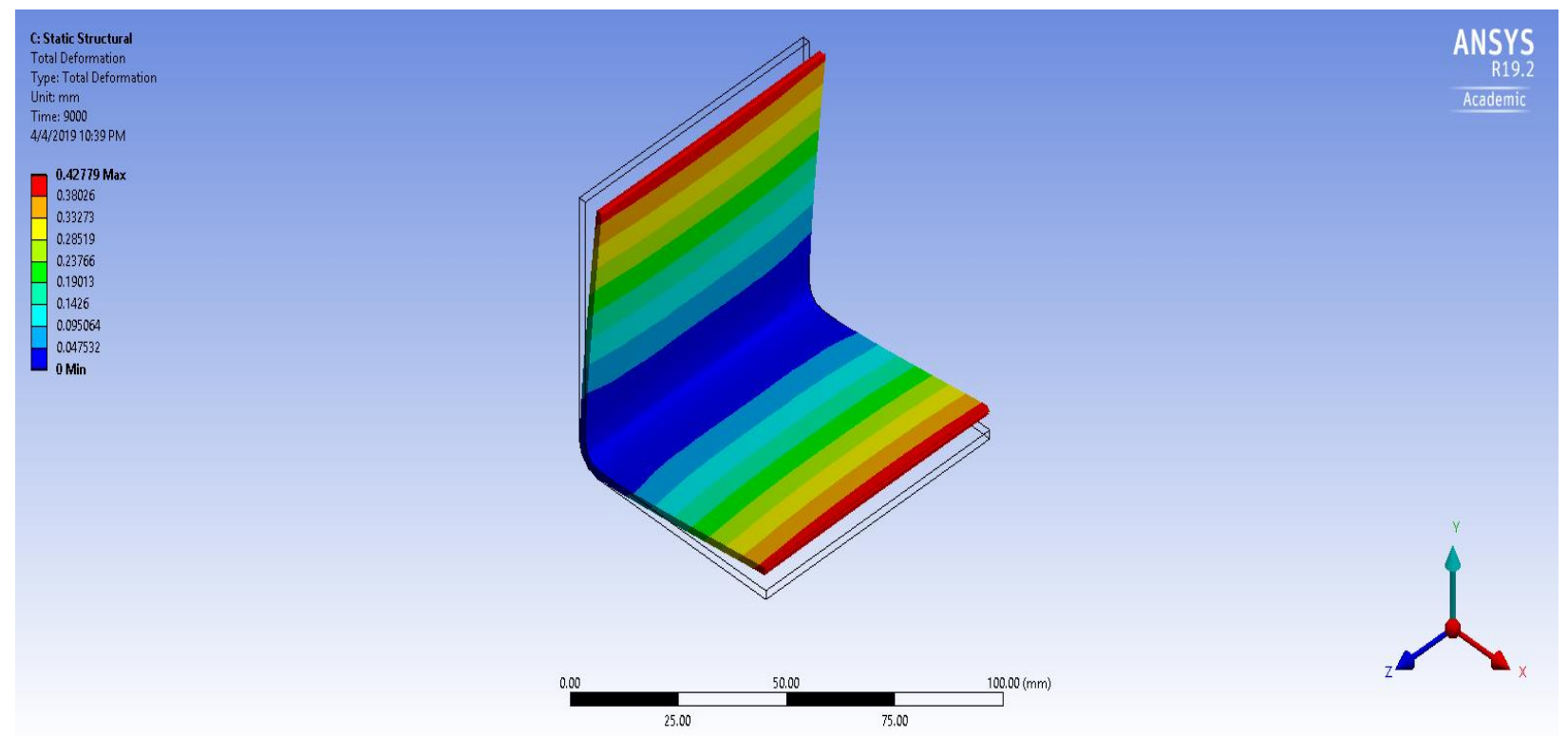

Figure 8. Spring-in for composite L-Shaped plate. 
The deformed L-shaped composite plate post curing is shown in figure 8 . The change in angle can clearly be observed. The values of the spring-in angle are obtained from the simulation results.

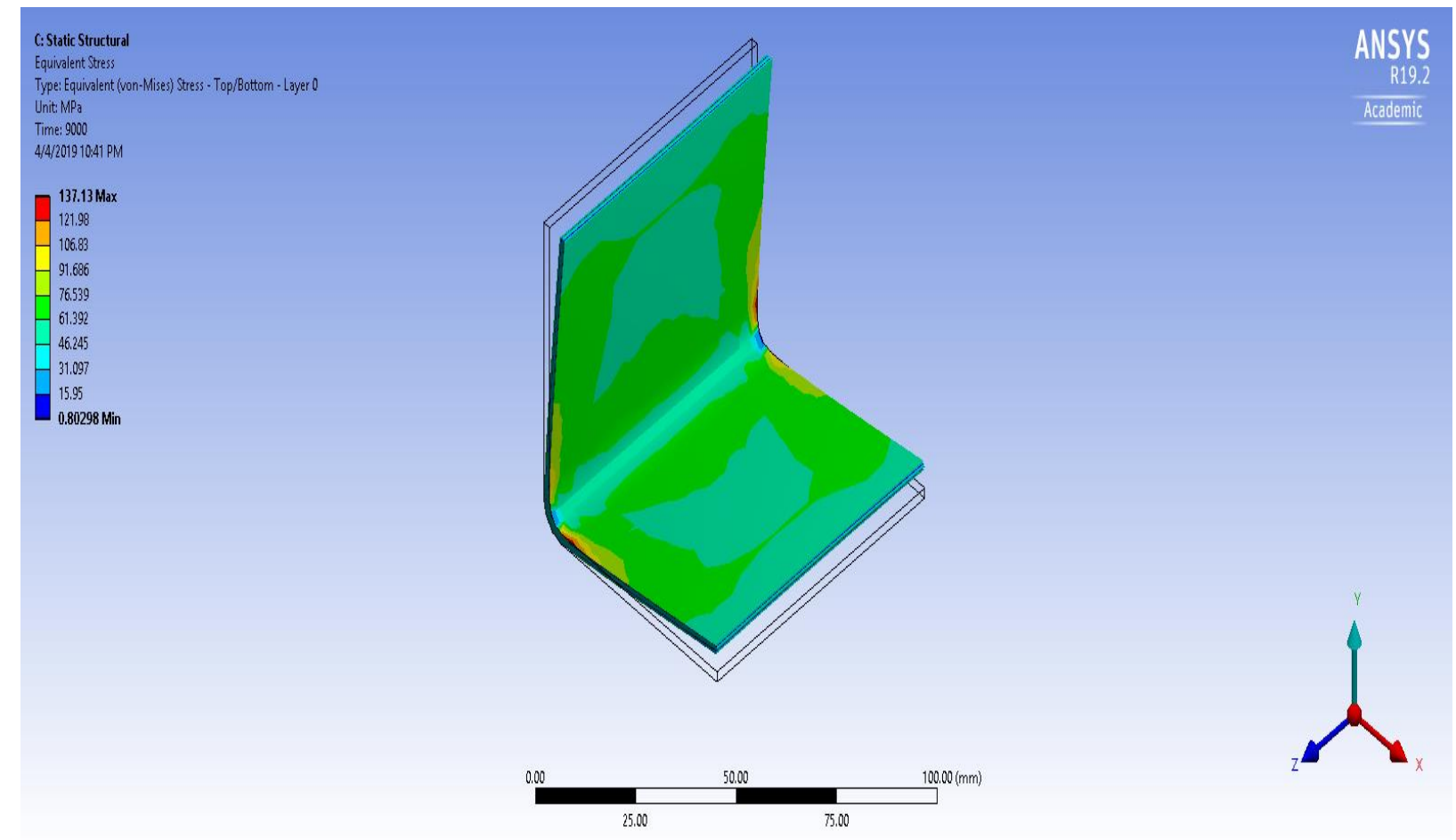

Figure 9. Residual Stresses generated in L-Shaped composite plate.

Since analytical calculation of process induced residual stresses is a time consuming and difficult process, obtaining the results for residual stresses through simulation is a comprehensive technique. The residual stresses induced owing to thermo-mechanical curing process are obtained in the form of contour plot of the composite part. Similar to the thermal module, structural module also provides the stresses induced in each individual ply. This pre-loading stresses over the composite parts can make the part weaker upon loading. Figure 9 is the contour of the residual stresses present in the composite part post deformation. The results for residual stresses are the stresses present in the part post deformation. Even if the part is deformed to get rid of stresses generated during the curing process, there are stresses present in the part post deformation. It is practically impossible to get rid of all the residual stresses as the stresses are trapped in the composite laminate during phase change of the resin. These stresses can be predicted and can be used as the initial stress condition for the part while the part is further analyzed for practical load conditions such as crash, impact and torsion or bending.

\section{COMPARISON WITH EXPERIMENTAL RESULTS}

The methodology presented here was validated by comparing the spring-in results obtained by simulation with the experimental results that were presented by various researchers whose work was reviewed [22]. The simulation results showed a similar trend as the 
experimental results as simulation was carried out for various parameters. In some cases, the spring-in values achieved from simulation results were found to be more accurate than the analytically calculated spring-in values.

LMAT [22] conducted experiments to verify effect of laminate thickness on spring-in angle. The trend in the LMAT results was similar to the simulation results obtained using the methodology presented here.

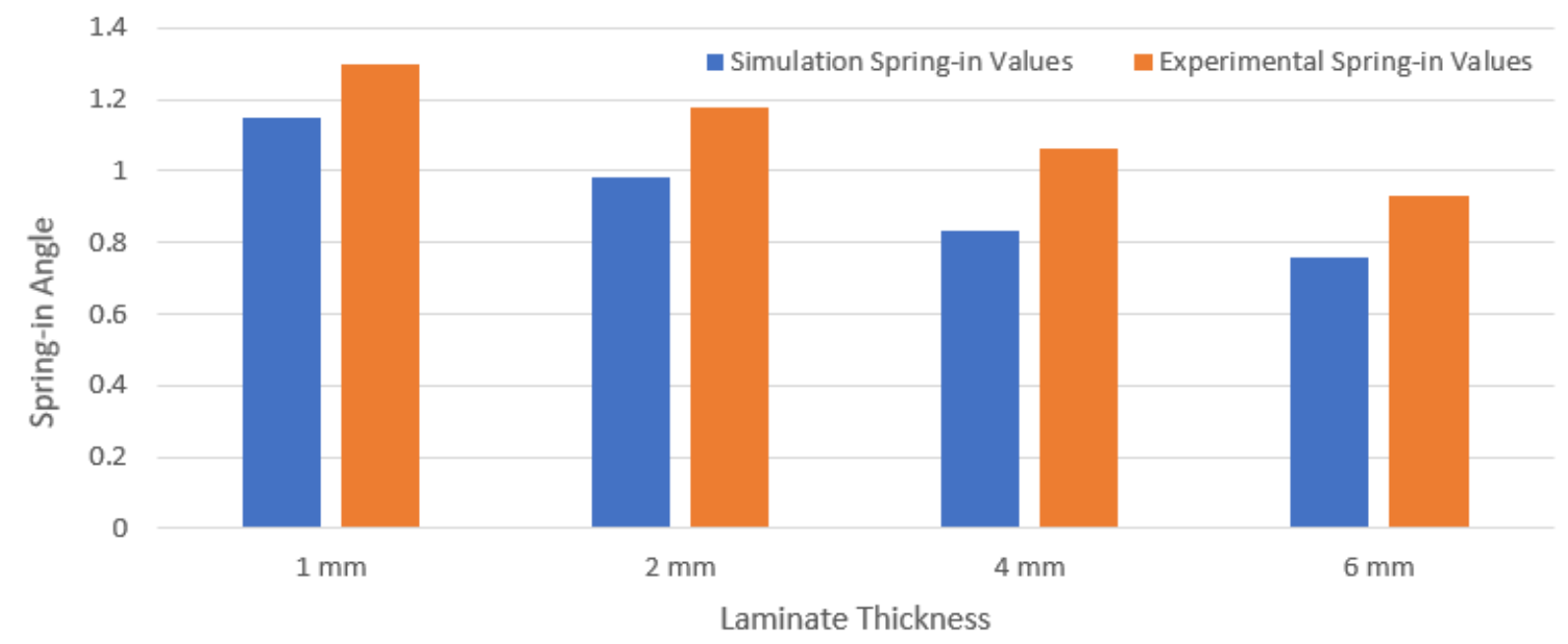

Figure 10. Plot of Spring-in vs Laminate Thickness.

Figure 10 demonstrations the comparison between the simulation spring-in values and experimental spring-in values for deferent laminate thickness. It is evident that as the laminate thickness increases the spring-in decreases. The difference between the simulation values and experimental values is due to the difference between the lay-up pattern used. Also effect of warpage and effect of tool-part interaction were neglected in this case while simulating the curing process and applying the structural loads. However, the pattern is similar which validates the accuracy of simulation results. The tests were conducted by LMAT to study the effect of cure shrinkage and CTE on the composite L-shaped plates.

Albert and Fernlund [2] presented a model where an analytical model was developed to calculate the spring-in for L-shaped and C-shaped composite parts. The simulation results provided results closer to experimental results presented in this particular study than the analytically obtained spring-in values. The comparison between analytical, Numerical (simulation) and experimental values of the spring in are presented in the table 3 . The simulation spring in value is closer to the experimental spring-in value than the analytical value. Figure 11 displays the simulation results of spring-in for a $\mathrm{C}$ shaped composite part.

TABLE 3. COMPARISON OF RESULTS WITH ANALYTICAL AND EXPERIMENTAL.

\begin{tabular}{|c|c|c|}
\hline Analytical Value of Spring-in & Numerical Value of Spring-in & $\begin{array}{c}\text { Experimental Value of Spring-in } \\
{[22]}\end{array}$ \\
\hline $0.90^{\circ}$ & $0.81^{\circ}$ & $0.78^{\circ}$ \\
\hline
\end{tabular}




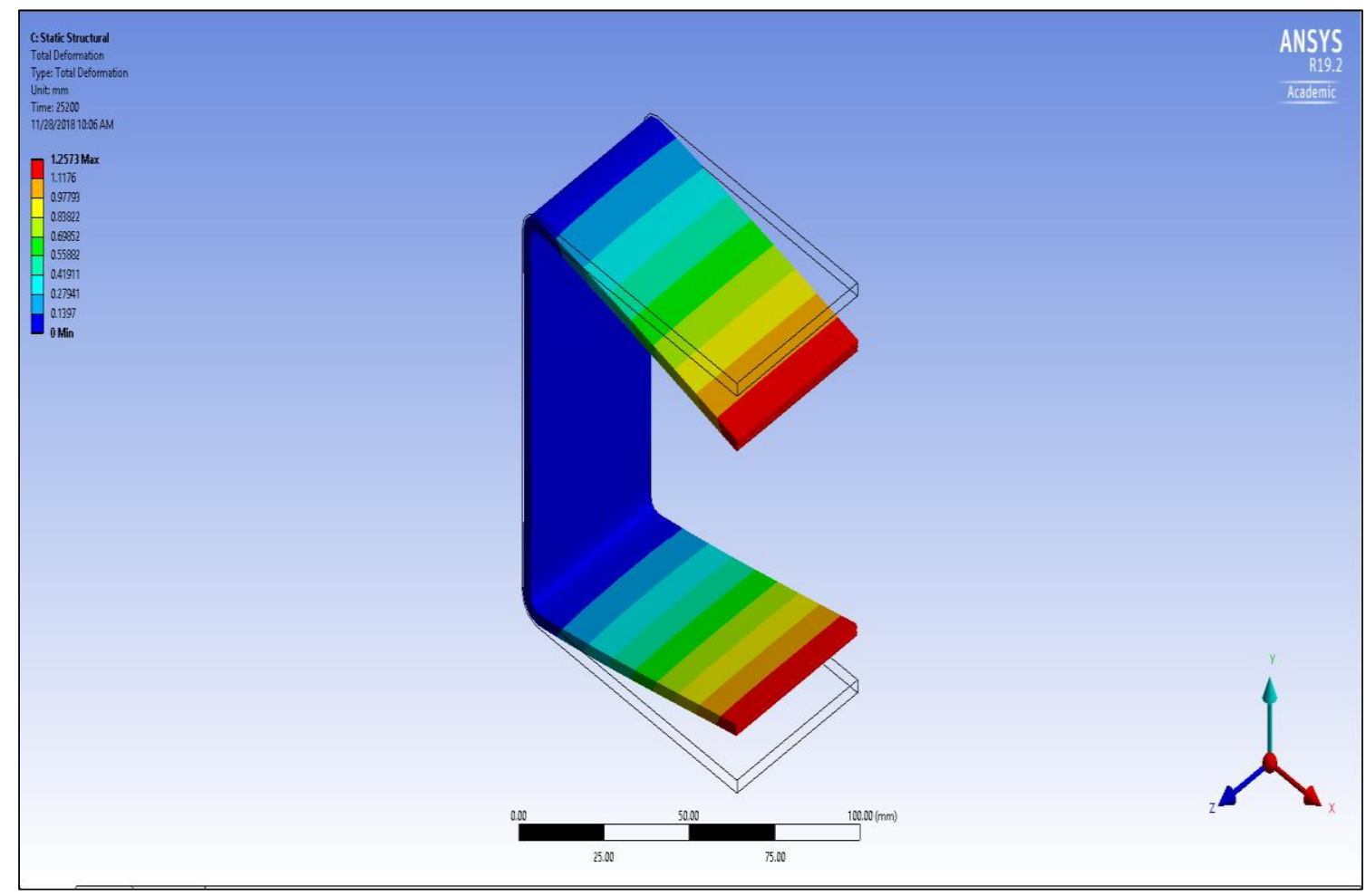

Figure 11. Simulation results of spring-in for composite C-Shaped plate.

\section{CONCLUSION}

This paper has provided a comprehensive methodology to predict the process induced shape distortions accurately and in less time. The ability to predict the final shape of the part before tool design is helpful in reducing the manufacturing cost. Although quite a few previous studies have emphasized using FEA for predicting the shape distortions, all the methods had several limitations. This study has proposed an approach to predict the final shape of the composite part not only for simple flat panels but also for complex composite parts using (ACCS).

Furthermore, the paper presented the thermal results for composite parts during cure. This helped in obtaining the results for all the thermal properties. While previous studies provided the structural results for spring-in this study presented both thermal and structural results. When compared with experimental results previously presented, the simulation results obtained here were observed to be more accurate than analytically calculated results.

The methodology developed here was found to be accurate, less time consuming and effective for further analysis of the composite parts for various practical purposes. This technique may open a new window in composite manufacturing with its higher accuracy. Also, the ability of using the deformed model for further mechanical analysis including static, cyclic and crash analysis. ACCS is a powerful tool which can be used to estimate the dimensional changes in the composite parts which are induced in the curing process. By simulating the entire curing process, it is possible to obtain the values of dimensional changes for different curing 
temperatures and curing cycles. Spring-in for different parameters can now also be analyzed easily.

\section{REFERENCES:}

1. Svanberg M, "Predictions of manufacturing induced shape distortions - high performance thermoset composites," PhD Thesis, Department of Applied Physics and Mechanical Engineering, Lule_a University of Technology, 2002.

2. Albert $\mathrm{C}$ and Fernlund G, "Spring-in and warpage of angled composite laminates," Composites Science and Technology, vol. 62, no. 14, pp. 1895-1912, 2002.

3. Zhang J, Ling Z, and Guan Z, "Analysis on factors influencing process-induced deformation for thermoset composites [j]," Acta Materiae Compositae Sinica, vol. 26, pp. 179-184, 2009.

4. Nelson R and Cairns D, "Prediction of dimensional changes in composite laminate during cure," 34th International SAMPE Symposium and Exhibition, 051989

5. Bapanapalli S K, Smith L V. "A linear finite element model to predict processing-induced distortion in FRP laminates[J]". Composites Part A: Applied Science and Manufacturing, 2005, 36(12): 1666-1674.n

6. Jain L. K, Hou M, Ye L. and W Mai. "Spring-in study of the aileron rib manufactures from advanced thermoplastic composite." Composites:Part A, 1998 29A, 973-979

7. Fernlund G, Poursartip A, Twigg G, and Albert C, "Residual stress, spring-in and warpage in autoclaved composite parts," Department of Metals and Materials Engineering, The University of British Columbia 3096350 Stores Rd., Vancouver, V6T 1Z4, Canada, 042010.

8. Fernlund G, Rahman N, Courdji R, Bresslauer M, Poursartip A, Willden K, and Nelson K. "Experimental and numerical study of the effect of cure cycle, tool surface, geometry and lay-up on the dimensional fidelity of autoclave-processed composite parts". Composites Part A, 33:341-351, 2002

9. Tavakol B, "Prediction of residual stresses and distortions of carbon fiber/ epoxy composites due to curing process," Master's Thesis, Department of Mechanical Engineering, Wichita state University, 2011.

10. Sprowitz T, Tessmer J, and Wille T, "Thermal aspects for composite structures- from manufacturing to inservice predictions," ICAS Secretariat -26th Congress of International Council of the Aeronautical Sciences 2008, ICAS 2008, vol. 2, 092008.

11. White SR, Hahn HT. "Cure cycle optimization for the reduction of processing-induced residual stresses in composite materials." Journal of Composite Materials 1993;27(14):1352-78

12. Lee W, Loos A, and Springer G, "Heat of reaction, degree of cure, and viscosity of Hercules 3501-6 resin," Journal of Composite Materials, vol. 16, 1982.

13. Pagano NJ. "Thickness expansion coefficients of composite laminates", Journal of Composite Materials 1974;8:310-2.

14. Carslaw HS, Jaeger JC. "Conduction of heat in solids". 2nd ed. Oxford: Oxford University Press; 1974.

15. Gigliotti M, Jacquemin F, and Vautrin A, "Internal stresses induced by cyclical hygrothermal conditions due to supersonic ight in laminated composite plates," AIAA J, vol. 42, 2004

16. Twigg GA, Poursartip A, Fernlund G. "Tool-part interaction in composites processing". Proceedings of the 13th International Conference on Composite Materials (ICCM13) 2001: paper 1192.

17. Twigg GA, Fernlund G, Poursartip A. "Measurement of tool-part interfacial stress during the processing of composite laminates", Proceedings of the 16th ASC Technical Conference 2001:85-96.

18. Bogetti $\mathrm{T}$ and Gillespie J, "Two-dimensional cure simulation of thick thermosetting composite," Journal of Composite Materials, vol. 25, 1991.

19. "Introduction of LMAT's ACCS ANSYS composite cure simulation toolbox," ANSYS Southpointe 2600 ANSYS Drive Canonsburg, PA 15317, U.S.A., 2017.

20. Patil H and Jeyakarthikeyan P, "Mesh convergence study and estimation of discretization error of hub in clutch disc with integration of ANSYS," vol. 402,pp. 12-65, Oct 2018

21. Khoun L, Centea T, and Hubert P, "Characterization methodology of thermoset resins for the processing of composite materials | case study: CYCOM 890 RTM Epoxy Resin," Journal of Composite Materials, vol. 44, no. 11, pp. 1397-1415, 2009.

22. Garstka T, "Numerical tool compensation and composite process optimization," A Presentation by LMAT Lean Manufacturing Assembly Technologies, 2017.

23. Keshavanarayana, S., Shahverdi, H., Kothare, A., Yang, C., Bingenheimer, J., The effect of node bond adhesive fillet on uniaxial in-plane responses of hexagonal honeycomb core, Compos. Struct. 175 (2017) 111-122. 
24. Yang, C., Shahverdi , H., Keshavanarayana, R., and Horner, A."An analytical approach to characterize uniaxial in-plane responses of commercial hexagonal honeycomb core under large deformations." Composite Structures 211 (2019): 100-111.

25. Moheimani, R., Sarayloo, R., Dalir, H. "Symmetrical and Antisymmetrical Sequenced Fibers with Epoxy Resin on Rectangular Reinforced Structures Under Axial Loading", 33st American society for composites (ASC) Technical Conference, 2018, doi:10.12783/asc33/25990. 